\title{
Trophic status of water and level of pollutants as measures of efficiency of water treatment regime at Kafr El-Shinawy drinking-water treatment plant, Damietta
}

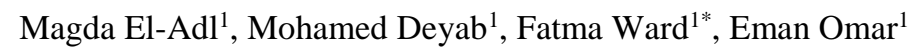 \\ 1 Department of Botany, Faculty of Science, Damietta University, New Damietta 34517, Egypt.
}

Received: 27 May 2020 /Accepted: 28 September 2020

*Corresponding author's E-mail: fatma2028@yahoo.com

\begin{abstract}
This work aims to study the seasonal fluctuation in physicochemical characteristics, trophic status, and some chemical and biological pollutants influencing phytoplankton diversity, and water quality in raw and treated water at Kafr El-Shinawy drinking-water treatment plant, Damietta - Egypt seasonally during 2018. Phytoplankton distribution was affected by the trophic status of water, level of pollutants, and physicochemical treatment processes (coagulation, flocculation and sedimentation) of water. The predominance of phytoplankton species, especially Aphanizomenon flos aquae (Cyanophyta), Gomphosphaeria lacustris (Cyanophyta), Microcystis aeruginosa (Cyanophyta), Nostoc punctiforme (Cyanophyta), Oscillatoria limnetica (Cyanophyta), Pediastrum simplex (Chlorophyta), and Melosira granulata (Bacillariophyta) in treated water was much less than in raw water. Trihalomethanes (THMs) levels in treated waters were higher than in raw water. On the contrary, lower concentrations of heavy metals were recorded in treated water. Phytoplankton cells exhibited a bioaccumulation capacity for heavy metals especially $\mathrm{Mn}, \mathrm{Zn}$, and Fe in both raw and treated water. Intracellular levels of microcystins were lower $(0.003-0.011 \mu \mathrm{g} L-1)$ whereas the extracellular levels were higher $(1.00-2.01 \mu \mathrm{g} \mathrm{L}-1)$ in treated water than raw water, and the former recorded the highest level in raw water during summer $(1.3 \mu \mathrm{g} \mathrm{L}-1)$. Hence, the levels of dissolved microcystins and THMs in treated water were higher than the allowable limit, especially during summer, the season of luxurious growth of Microcystis species.
\end{abstract}

Keywords: Drinking water; Microcystins; Phytoplankton; Physicochemical analyses; Trophic state; Water quality.

\section{Introduction}

Water pollution has become one of the most important environmental problems worldwide. Water treatment plants, mainly aims to improve the quality of water to make it appropriate for drinking and human consumption. Water treatment involves some physical processes such as settling and filtration, chemical processes such as disinfection and coagulation, in addition to biological processes such as slow sand filtration. The nutrient state and physicochemical characteristics of water have an important role in the fluctuations of the phytoplankton community 
(Kumar and Sahu 2012). One of the natural bioindicators of water quality is phytoplankton occurrence and abundance due to their sensitivity, nutrient availability, and environmental conditions (e.g. water temperature and level of salinity (Manickam et al. 2012). Water quality can be assessed through both qualitative and quantitative monitoring of phytoplankton as they are the primary producers representing the first trophic level in the food chain. The occurrence of harmful cyanobacterial blooms in drinking water represents a serious threat to human and animal health worldwide. Cyanobacterial growth adversely- affects odor, taste, and color of water as some of these cyanobacteria produce potent toxins called cyanotoxins. There are more than 100 identified variants of cyanotoxins that are commonly produced by the genera, Microcystis, Anabaena, Aphanizomenon, Fischerella, Planktothrix, Anabaenopsis, Aphanocapsa, Cylindrospermopsis, Gleotrichia, Gomphosphaeria, Hapalosiphon, Nodularia, Nostoc, Oscillatoria, Phormidium, Pseudanabaena, and Synechococcus (Chorus et al. 2000; Mohamed and Carmichael 2000; Vesterkvist et al. 2012; Paerl and Otten 2013). Although cyanobacterial cells can survive in water for long periods due to their ability to form thickwalled resting cells, the production of cyanotoxins is affected by several environmental conditions such as temperature, salinity, irradiance, and nutrients (Visser et al. 2016).

As a result of chlorine addition during treatment of drinking water, Trihalomethanes (THMs) including chloroform, dichlorobromomethane, and dibromochloromethane are produced as byproducts. THMs have short-term and long-term hazardous effects on human health. Essential serious problem arises from the increase in THMs during water transport by the water pipe network. The present work aims to study the effect of seasonal changes in physicochemical characteristics and the trophic status of water as well as the levels of pollutants, and microbial toxins on water quality and phytoplankton diversity at the input and output sites of a drinkingwater treatment plant at Damietta.

\section{Material and methods}

\section{Sampling sites}

The study site was Kafr El-Shinawy drinking water treatment plant that is situated at $31^{\circ} 41.816^{\prime}$ $\mathrm{N}$ and $31^{\circ} 17.325^{\prime}$ E. Water samples were collected seasonally (at three month intervals) in glass bottles from both the intake and output sites of Kafr El-Shinawy water treatment plant during 2018 to determine the phytoplankton composition in relation to physicochemical properties, trophic status and levels of pollutants of the native and treated water.

\section{Physicochemical properties of water}

Temperature, turbidity, $\mathrm{pH}$, and electrical conductivity (EC) were measured in the field. Temperature and $\mathrm{pH}$ of water samples were measured using the laboratory glass thermometer and a pH meter (model HI 8314, Hanna Instruments Ltd), respectively. Water turbidity was measured directly using Hanna instrument microprocessor turbidity meter. Water EC was measured using Jenway conductivity meter model 470. Total alkalinity, dissolved oxygen (DO), biochemical oxygen demand (BOD), silica, ammonia, nitrite, nitrate, total nitrogen, and orthophosphate were estimated in the laboratory according to APHA (1996). The total phosphorus in water samples was determined according to Grasshoff (1975).

The heavy metals, iron, manganese, zinc, copper, chromium, cobalt, cadmium, nickel, and lead in water samples was assayed in water by using a Perkin-Elmer - 2380 atomic absorption spectrophotometer as described by Sudharsan et al. (2012). All physicochemical analyses of water samples were triplicated.

Trihalomethane compounds in native and treated water were estimated according to U.S. EPA Method 551.1 (1995).

\section{Phytoplankton composition}

Raw and treated water samples were collected seasonally for microscopic examination using a conical bolting nylon net of $0.069 \mathrm{~mm}$ mesh and a mouth diameter of $35 \mathrm{~cm}$ with the help of an outrigger canoe. The samples were filtered through fine mesh nylon and fixed in Lugol's solution and $4 \%$ formalin and algal cells were enumerated using an inverted light microscope (Sharma 2002). Phytoplankton identification was performed with reference to Botes (2003), Krammer and Lange-Bertalot (1986), and Tikkanen (1986) using an EXACTA+OPTECH GmbH light microscope (Model B3) - Code 
K7161, Germany

\section{Extraction and estimation of intracellular and extracellular microcystins}

To determine the intracellular (particulate) and extracellular microcystins in raw and treated water, subsamples $(250 \mathrm{ml})$ were filtered through a $0.45 \mu \mathrm{m}$ cellulose filter (Whatman, UK). The filtrate was kept frozen to be used for extracellular (dissolved) microcystins. The residue with trapped cells was frozen, extracted twice in $80 \%$ methanol and centrifuged at $10,000 \times g$ for $10 \mathrm{~min}$. The supernatants were pooled together, and the organic solvent was blown with sterilized air. The aqueous fraction remaining after removing the organic solvent was filtered through GF/C filter paper and stored frozen until analysis. Concentrations of extracellular and intracellular microcystins were determined by Highperformance liquid chromatography (HPLC) (Column, Nucleosil $5 \mathrm{C} 1 \sim(150 \times 4.6 \mathrm{~mm})$. Solvent system was: methanol - $0.05 \mathrm{M}$ phosphate buffer $(\mathrm{pH} 3)(58: 42)$. The flow rate was $1 \mathrm{ml} / \mathrm{min}$. Detection was at $238 \mathrm{~nm}$ (Harada et al. 1990).

\section{Biochemical composition of the predominant phytoplankton in raw and treated waters.}

Proteins and lipids (\% DW) of predominant species were estimated during winter and summer according to AOAC (2000) and carbohydrates were estimated spectrophotometry according to Dubois et al. (1956).

Chlorophyll-a - as a measure of phytoplankton biomass - was determined spectrophotometrically in $90 \%$ acetone extract of raw and treated waters according to Metzener et al. (1965) using the following equations:

Chlorophyll-a = $11.78\left(\mathrm{~A}_{663}\right)-2.29\left(\mathrm{~A}_{647}\right)$ Concentrations of the heavy metals (Fe, Mn, Zn, $\mathrm{Cu}, \mathrm{Cr}, \mathrm{Co}, \mathrm{Cd}, \mathrm{Ni}$, and $\mathrm{Pb}$ ) in phytoplankton cells from raw and treated water were estimated seasonally by using a Perkin-Elmer -2380 atomic absorption spectrophotometer as described by Sudharsan et al. (2012).

\section{Statistical analyses}

Data were analyzed using two-way ANOVA, followed by mean separation according to the
Duncan's multiple range test at $\mathrm{P}<0.05$. Twotailed Pearson product-moment correlation was performed to examine the relationship between all physicochemical parameters, phytoplankton diversity, and microcystin concentrations. Statistical analysis was done using SPSS version 22.

\section{Results}

\section{Physicochemical properties of water}

The effect of the main factors (water treatment and season) and their interaction was significant $(\mathrm{P}<$ 0.05 ) on most physicochemical parameters of water at Kafr El-Shinawy treatment plant as shown in Table 1.

Table 2 summarizes the physic-chemical characteristics of the raw and treated water at Kafr El-Shinawy treatment plant during 2018. The results showed significant variations in temperatures of both raw and treated water ranging from $17.95 \pm 2.48{ }^{\circ} \mathrm{C}$ during winter to $31.0 \pm 2.44^{\circ} \mathrm{C}$ during summer, with optimum for phytoplankton growth during autumn and spring $\left(25.0-26.0^{\circ} \mathrm{C}\right)$. Turbidity recorded higher values in raw water than that in treated water with lower values in both raw and treated water during winter. Water $\mathrm{pH}$ value was generally in the alkaline side and ranged between 7.76 and 8.51 with lower values in treated than raw water. In both raw and treated water, EC was highest in summer (Table 2 ). Throughout the four seasons, raw water recorded higher alkalinity compared with treated water. Dissolved Oxygen (DO) showed higher concentrations in treated than raw water 6.67 and $7.57 \mathrm{mg} \mathrm{L}^{-1}$, respectively (Table 2). It is also observed that DO in raw water increased by decreasing water temperature. On the contrary, BOD of both raw and treated water increased with increasing water temperature. Silica concentrations were higher in treated than raw water and approached their maxima during winter. Concentrations of both ortho-P and total- $\mathrm{P}$ were higher in raw water than that in treated water, with limited seasonal variability.

The effect of the main factors (water treatment and season) and their interaction on heavy metals concentrations of water was significant (Table 3 ). 
Table 1. Two- way ANOVA showing the effect of the main factors (water treatment and season) and their interaction on physicochemical parameters of water at Kafr El-Shinawy drinking-water treatment plant - Damietta.

\begin{tabular}{|c|c|c|c|c|c|c|c|}
\hline $\begin{array}{c}\text { Variable and treatment of } \\
\text { variation }\end{array}$ & df & $\mathrm{F}$ & $\mathrm{P}$ & $\begin{array}{c}\text { Variable and treatment of } \\
\text { variation }\end{array}$ & df & $\mathrm{F}$ & $\mathrm{P}$ \\
\hline Temperature & & & & Silica & & & \\
\hline Water treatment & 1 & 1216.89 & 0.000 & Water treatment & 1 & 381.045 & 0.000 \\
\hline Season & 3 & 23956.6 & 0.000 & Season & 3 & 576.301 & 0.000 \\
\hline $\begin{array}{l}\text { Water treatment } \times \text { season } \\
\text { Turbidity }\end{array}$ & 3 & 75.704 & 0.000 & $\begin{array}{l}\text { Water treatment } \times \text { season } \\
\text { Ammonia }\end{array}$ & 3 & 208.962 & 0.000 \\
\hline Water treatment & 1 & 16419.6 & 0.000 & Water treatment & 1 & 13268.6 & 0.000 \\
\hline Season & 3 & 306.935 & 0.000 & Season & 3 & 79.881 & 0.000 \\
\hline $\begin{array}{l}\text { Water treatment } \times \text { season } \\
\text { pH }\end{array}$ & 3 & 113.231 & 0.000 & $\begin{array}{l}\text { Water treatment } \times \text { season } \\
\text { Nitrite }\end{array}$ & 3 & 79.881 & 0.000 \\
\hline Water treatment & 1 & 108.926 & 0.000 & Water treatment & 1 & 1216.00 & 0.000 \\
\hline Season & 3 & 30.894 & 0.000 & Season & 3 & 59.368 & 0.000 \\
\hline $\begin{array}{l}\text { Water treatment } \times \text { season } \\
\text { Conductivity }\end{array}$ & 3 & 1.6570 & 0.216 & $\begin{array}{l}\text { Water treatment } \times \text { season } \\
\text { Nitrate }\end{array}$ & 3 & 59.368 & 0.000 \\
\hline Water treatment & 1 & 0.1560 & 0.698 & Water treatment & 1 & 9130.08 & 0.000 \\
\hline Season & 3 & 365.173 & 0.000 & Season & 3 & 20.306 & 0.000 \\
\hline $\begin{array}{l}\text { Water treatment } \times \text { season } \\
\text { Total alkalinity }\end{array}$ & 3 & 0.7250 & 0.552 & $\begin{array}{l}\text { Water treatment } \times \text { season } \\
\text { Total-N }\end{array}$ & 3 & 20.306 & 0.000 \\
\hline Water treatment & 1 & 940.612 & 0.000 & Water treatment & 1 & 92852.0 & 0.000 \\
\hline Season & 3 & 1681.57 & 0.000 & Season & 3 & 22.522 & 0.000 \\
\hline $\begin{array}{l}\text { Water treatment } \times \text { season } \\
\text { DO }\end{array}$ & 3 & 9.2190 & 0.001 & $\begin{array}{l}\text { Water treatment } \times \text { season } \\
\text { Ortho-P }\end{array}$ & 3 & 22.522 & 0.000 \\
\hline Water treatment & 1 & 1315.09 & 0.000 & Water treatment & 1 & 13572.3 & 0.000 \\
\hline Season & 3 & 181.031 & 0.000 & Season & 3 & 4.250 & 0.022 \\
\hline $\begin{array}{l}\text { Water treatment } \times \text { season } \\
\text { BOD }\end{array}$ & 3 & 134.635 & 0.000 & $\begin{array}{l}\text { Water treatment } \times \text { season } \\
\text { Total-P }\end{array}$ & 3 & 2.250 & 0.122 \\
\hline Water treatment & 1 & 13489.7 & 0.000 & Water treatment & 1 & 3864.39 & 0.000 \\
\hline Season & 3 & 988.937 & 0.000 & Season & 3 & 6.556 & 0.004 \\
\hline Water treatment $\times$ season & 3 & 224.678 & 0.000 & Water treatment $\times$ season & 3 & 9.912 & 0.001 \\
\hline
\end{tabular}

The effect of water treatment was stronger (with higher $\mathrm{F}$ ratio) than that of season for all determined heavy metals that decreased in treated water than that in raw water. Levels of all the measured heavy metals especially $\mathrm{Mn}, \mathrm{Zn}$, and $\mathrm{Fe}$ were higher in phytoplankton cells than that in raw water and treated water (Table 4). Correlation between heavy metals and other physicochemical parameters of both raw and treated water is presented in Table 5 .

The effect of the main factors (water teatment and season) and their interaction on THMs in raw and treated water was significant $(\mathrm{P}<0.05)$ as shown in Table 6. The effect of water treatment was stronger (with higher F ratio) than that of a season for all tested THMs. The present results showed that high values of THMs in water were during summer, whereas low concentrations were during winter, with an increase in treated water. The water treatment exhibited its maximum efficiency in winter. THMs specification shows that their presence in both raw and treated water was in the order: chloroform > dichlorobromomethane > dibromochloromethane.

As shown in Fig. 1, chloroform concentrations in raw and treated water was in the range of $2.79-$ $17.43 \mathrm{mg} \mathrm{L}^{-1}$ and 18.42 - $69.75 \mathrm{mg} \mathrm{L}^{-1}$, respectively. The results showed significant variations in dichlorobromomethane in raw and treated water $(\mathrm{P}<0.05)$ ranging from $1.52 \mathrm{mg} \mathrm{L}^{-1}$ (in raw water during winter) to $48.36 \mathrm{mg} \mathrm{L}^{-1}$ (in treated water during summer). The maximum concentration of dibromochloromethane was $25.98 \mathrm{mg} \mathrm{L}^{-1}$ in treated water during summer. Moreover, THMs correlated negatively with nutrients in both the native and treated water. 
Table 2. Seasonally variations in physicochemical characteristics (Mean $\pm S E, n=3$ ) of raw and treated waters of Kafr El-Shinawy drinking-water treatment plant - Damietta.

\begin{tabular}{|c|c|c|c|c|c|}
\hline \multirow{2}{*}{ Water characteristic } & \multirow{2}{*}{ Water treatment } & \multicolumn{4}{|c|}{ Season } \\
\hline & & Winter & Spring & Summer & Autumn \\
\hline \multirow{2}{*}{ Temperature $\left({ }^{\circ} \mathrm{C}\right)$} & Raw water & $17.95 \pm 2.48^{b}$ & $25.5 \pm 2.54^{\mathrm{cd}}$ & $31.0 \pm 2.44^{\mathrm{g}}$ & $26.0 \pm 2.69^{\mathrm{e}}$ \\
\hline & Treated water & $16.1 \pm 1.59^{\mathrm{a}}$ & $25.0 \pm 2.58^{c}$ & $29.4 \pm 1.79^{\mathrm{f}}$ & $25.1 \pm 2.51^{\mathrm{c}}$ \\
\hline \multirow{2}{*}{ Turbidity (NTU) } & Raw water & $4.30 \pm 0.38^{\mathrm{d}}$ & $6.01 \pm 0.57^{\mathrm{f}}$ & $6.00 \pm 0.57^{\mathrm{f}}$ & $5.04 \pm 0.50^{\mathrm{e}}$ \\
\hline & Treated water & $1.27 \pm 0.12^{\mathrm{a}}$ & $1.50 \pm 0.15^{\mathrm{b}}$ & $1.86 \pm 0.18^{\mathrm{c}}$ & $1.34 \pm 0.13^{\mathrm{a}}$ \\
\hline \multirow[b]{2}{*}{$\mathrm{pH}$} & Raw water & $7.76 \pm 0.91^{\mathrm{d}}$ & $8.12 \pm 0.81^{g}$ & $8.51 \pm 0.75^{\mathrm{h}}$ & $7.90 \pm 0.79^{\mathrm{ef}}$ \\
\hline & Treated water & $7.34 \pm 0.72^{\mathrm{a}}$ & $7.58 \pm 0.75^{\mathrm{bc}}$ & $7.86 \pm 0.78^{\mathrm{e}}$ & $7.53 \pm 0.74^{b}$ \\
\hline \multirow{2}{*}{ Conductivity $\left(\mathrm{dS} \mathrm{m}^{-1}\right)$} & Raw water & $540.0 \pm 35.0^{\mathrm{a}}$ & $600.7 \pm 60.1^{\mathrm{e}}$ & $750.0 \pm 73.3^{f}$ & $550.3 \pm 34.7^{b}$ \\
\hline & Treated water & $550.0 \pm 51.1^{\mathrm{b}}$ & $590.3 \pm 58.1^{\mathrm{d}}$ & $755.0 \pm 73.9^{\mathrm{g}}$ & $554.0 \pm 54.5^{\mathrm{bc}}$ \\
\hline \multirow{2}{*}{ Total Alk. $\left(\mathrm{mg} \mathrm{L}^{-1}\right)$} & Raw water & $147.0 \pm 13.8^{c}$ & $162.0 \pm 16.0^{\mathrm{e}}$ & $174.0 \pm 17.3^{\mathrm{g}}$ & $147.3 \pm 14.4^{\mathrm{bc}}$ \\
\hline & Treated water & $136.1 \pm 13.1^{\mathrm{a}}$ & $151.3 \pm 14.8^{\mathrm{d}}$ & $164.7 \pm 16.3^{\mathrm{ef}}$ & $140.3 \pm 14.0^{\mathrm{b}}$ \\
\hline \multirow{2}{*}{$\mathrm{DO}\left(\mathrm{mg} \mathrm{L}^{-1}\right)$} & Raw water & $6.83 \pm 0.61^{\mathrm{e}}$ & $6.09 \pm 0.58^{b}$ & $5.10 \pm 0.50^{\mathrm{a}}$ & $6.20 \pm 0.59^{c}$ \\
\hline & Treated water & $7.10 \pm 0.71^{\mathrm{g}}$ & $7.57 \pm 0.74^{\mathrm{h}}$ & $6.67 \pm 0.67^{\mathrm{d}}$ & $7.00 \pm 0.64^{\mathrm{f}}$ \\
\hline \multirow{2}{*}{$\mathrm{BOD}\left(\mathrm{mg} \mathrm{L}^{-1}\right)$} & Raw water & $2.71 \pm 0.27^{\mathrm{e}}$ & $2.99 \pm 0.29^{f}$ & $3.81 \pm 0.32^{\mathrm{h}}$ & $3.50 \pm 0.35^{\mathrm{g}}$ \\
\hline & Treated water & $1.51 \pm 0.15^{\mathrm{a}}$ & $2.00 \pm 0.18^{c}$ & $2.23 \pm 0.22^{\mathrm{d}}$ & $1.71 \pm 0.17^{\mathrm{b}}$ \\
\hline \multirow{2}{*}{ Silica $\left(\mathrm{mg} \mathrm{L}^{-1}\right)$} & Raw water & $2.51 \pm 0.52^{\mathrm{c}}$ & $3.10 \pm 0.30^{\mathrm{d}}$ & $3.60 \pm 0.31^{\mathrm{f}}$ & $2.23 \pm 0.22^{\mathrm{a}}$ \\
\hline & Treated water & $4.00 \pm 0.34^{\mathrm{h}}$ & $3.30 \pm 0.32^{\mathrm{e}}$ & $3.67 \pm 0.35^{\mathrm{fg}}$ & $2.33 \pm 0.22^{\mathrm{b}}$ \\
\hline \multirow{2}{*}{ Ammonia $\left(\mathrm{mg} \mathrm{L}^{-1}\right)$} & Raw water & $0.42 \pm 0.038^{f}$ & $0.35 \pm 0.034^{\mathrm{d}}$ & $0.28 \pm 0.021^{\mathrm{c}}$ & $0.39 \pm 0.038^{\mathrm{e}}$ \\
\hline & Treated water & $0.02 \pm 0.001^{\mathrm{ab}}$ & $0.01 \pm 0.001^{\mathrm{a}}$ & $0.01 \pm 0.001^{\mathrm{a}}$ & $0.01 \pm 0.001^{\mathrm{a}}$ \\
\hline \multirow{2}{*}{ Nitrite $\left(\mathrm{mg} \mathrm{L}^{-1}\right)$} & Raw water & $0.20 \pm 0.015^{\mathrm{g}}$ & $0.09 \pm 0.008^{\mathrm{de}}$ & $0.08 \pm 0.007^{\mathrm{d}}$ & $0.12 \pm 0.011^{\mathrm{f}}$ \\
\hline & Treated water & $0.05 \pm 0.003^{\mathrm{c}}$ & $0.01 \pm 0.002^{\mathrm{a}}$ & $0.01 \pm 0.002^{\mathrm{a}}$ & $0.03 \pm 0.004^{\mathrm{b}}$ \\
\hline \multirow{2}{*}{ Nitrate $\left(\mathrm{mg} \mathrm{L}^{-1}\right)$} & Raw water & $0.31 \pm 0.031^{\mathrm{h}}$ & $0.27 \pm 0.026^{\mathrm{g}}$ & $0.24 \pm 0.023^{\mathrm{e}}$ & $0.28 \pm 0.028^{f}$ \\
\hline & Treated water & $0.20 \pm 0.003^{\mathrm{d}}$ & $0.15 \pm 0.002^{\mathrm{c}}$ & $0.09 \pm 0.002^{\mathrm{ab}}$ & $0.08 \pm 0.002^{\mathrm{a}}$ \\
\hline \multirow{2}{*}{ Total -N (mg L $\left.{ }^{-1}\right)$} & Raw water & $2.72 \pm 0.270^{\mathrm{d}}$ & $2.74 \pm 0.272^{\mathrm{de}}$ & $2.91 \pm 0.223^{g}$ & $2.76 \pm 0.275^{\text {def }}$ \\
\hline & Treated water & $0.19 \pm 0.009^{c}$ & $0.11 \pm 0.003^{\mathrm{a}}$ & $0.20 \pm 0.004^{c}$ & $0.15 \pm 0.004^{\mathrm{b}}$ \\
\hline \multirow{2}{*}{ Ortho $-\mathrm{P}\left(\mathrm{mg} \mathrm{L}^{-1}\right)$} & Raw water & $0.021 \pm 0.005^{\mathrm{cd}}$ & $0.020 \pm 0.002^{\mathrm{c}}$ & $0.020 \pm 0.002^{\mathrm{c}}$ & $0.020 \pm 0.002^{\mathrm{c}}$ \\
\hline & Treated water & $0.006 \pm 0.0001^{\mathrm{ab}}$ & $0.005 \pm 0.0001^{\mathrm{a}}$ & $0.006 \pm 0.0001^{\mathrm{ab}}$ & $0.006 \pm 0.0001^{\mathrm{ab}}$ \\
\hline \multirow{2}{*}{ Total - P $\left(\mathrm{mg} \mathrm{L}^{-1}\right)$} & Raw water & $1.70 \pm 0.13^{\mathrm{d}}$ & $1.82 \pm 0.178^{\mathrm{ef}}$ & $1.99 \pm 0.21^{\mathrm{g}}$ & $1.80 \pm 0.22^{\mathrm{e}}$ \\
\hline & Treated water & $0.50 \pm 0.056^{\mathrm{b}}$ & $0.50 \pm 0.055^{\mathrm{b}}$ & $0.52 \pm 0.057^{\mathrm{bc}}$ & $0.45 \pm 0.050^{\mathrm{a}}$ \\
\hline
\end{tabular}

Table 3. Two- way ANOVA showing the effect of the main factors (water teatment and season) and their interaction on heavy metals concentrations of raw and treated waters at Kafr El-Shinawy drinking-water treatment plant - Damietta.

\begin{tabular}{|c|c|c|c|c|c|c|c|}
\hline $\begin{array}{c}\text { Variable and treatment of } \\
\text { variation }\end{array}$ & df & $\mathrm{F}$ & $\mathrm{P}$ & $\begin{array}{c}\text { Variable and treatment of } \\
\text { variation }\end{array}$ & $\mathrm{df}$ & $\mathrm{F}$ & $\mathrm{P}$ \\
\hline $\mathrm{Fe}$ & & & & Co & & & \\
\hline Water treatment & 1 & 94848.0 & 0.000 & Water treatment & 1 & 1366561 & 0.000 \\
\hline Season & 3 & 118.327 & 0.000 & Season & 3 & 84521.0 & 0.000 \\
\hline Water treatment $\times$ season & 3 & 130.171 & 0.000 & Water treatment $\times$ season & 3 & 70721.0 & 0.000 \\
\hline Mn & & & & Cd & & & \\
\hline Water treatment & 1 & 3910.10 & 0.000 & Water treatment & 1 & 2726112 & 0.000 \\
\hline Season & 3 & 51.391 & 0.000 & Season & 3 & 12703.2 & 0.000 \\
\hline $\begin{array}{l}\text { Water treatment } \times \text { season } \\
\text { Zn }\end{array}$ & 3 & 9.1420 & 0.001 & $\begin{array}{l}\text { Water treatment } \times \text { season } \\
\mathbf{N i}\end{array}$ & 3 & 12503.2 & 0.000 \\
\hline Water treatment & 1 & 41538.8 & 0.000 & Water treatment & 1 & 84807.7 & 0.000 \\
\hline Season & 3 & 97.835 & 0.000 & Season & 3 & 2718.66 & 0.000 \\
\hline $\begin{array}{l}\text { Water treatment } \times \text { season } \\
\mathbf{C u}\end{array}$ & 3 & 365.482 & 0.000 & $\begin{array}{l}\text { Water treatment } \times \text { season } \\
\mathbf{P b}\end{array}$ & 3 & 2009.74 & 0.000 \\
\hline Water treatment & 1 & 3087049 & 0.000 & Water treatment & 1 & 2910436 & 0.000 \\
\hline Season & 3 & 135273 & 0.000 & Season & 3 & 5660.00 & 0.000 \\
\hline $\begin{array}{l}\text { Water treatment } \times \text { season } \\
\mathbf{C r}\end{array}$ & 3 & 75513.0 & 0.000 & Water treatment $\times$ season & 3 & 6620.00 & 0.000 \\
\hline Water treatment & 1 & 15987.0 & 0.000 & & & & \\
\hline Season & 3 & 677.667 & 0.000 & & & & \\
\hline Water treatment $\times$ season & 3 & 197.667 & 0.000 & & & & \\
\hline
\end{tabular}


Table 4. Seasonally variations in concentrations of some heavy metals (Mean $\pm S E, n=3$ ) in raw and treated waters, and phytoplankton cells Kafr El-Shinawy drinking-water treatment plant - Damietta.

\begin{tabular}{|c|c|c|c|c|c|}
\hline \multirow{2}{*}{ Heavy metal } & \multirow{2}{*}{ Treatment } & \multicolumn{4}{|c|}{ Season } \\
\hline & & Winter & Spring & Summer & Autumn \\
\hline & & \multicolumn{4}{|c|}{ Water } \\
\hline \multirow{2}{*}{$\mathrm{Fe}\left(\mathrm{mg} \mathrm{L}^{-1}\right)$} & Raw & $0.097 \pm 0.0048^{\mathrm{de}}$ & $0.120 \pm 0.0060^{\mathrm{g}}$ & $0.100 \pm 0.0050^{\mathrm{def}}$ & $0.094 \pm 0.0047^{\mathrm{d}}$ \\
\hline & Treated & $0.060 \pm 0.0030^{c}$ & $0.051 \pm 0.0026^{\mathrm{b}}$ & $0.040 \pm 0.0020^{\mathrm{a}}$ & $0.060 \pm 0.0030^{\mathrm{c}}$ \\
\hline \multirow{2}{*}{$\operatorname{Mn}\left(\mathrm{mg} \mathrm{L}^{-1}\right)$} & Raw & $0.067 \pm 0.0034^{\mathrm{g}}$ & $0.057 \pm 0.0029^{\mathrm{e}}$ & $0.070 \pm 0.0035^{\mathrm{h}}$ & $0.061 \pm 0.0031^{\mathrm{f}}$ \\
\hline & Treated & $0.014 \pm 0.0007^{\mathrm{ab}}$ & $0.020 \pm 0.0010^{\mathrm{c}}$ & $0.030 \pm 0.0015^{\mathrm{d}}$ & $0.010 \pm 0.0005^{\mathrm{a}}$ \\
\hline \multirow{2}{*}{$\mathrm{Zn}\left(\mathrm{mg} \mathrm{L}^{-1}\right)$} & Raw & $0.037 \pm 0.0019^{\mathrm{g}}$ & $0.032 \pm 0.0016^{\mathrm{def}}$ & $0.030 \pm 0.0015^{\mathrm{de}}$ & $0.029 \pm 0.0015^{\mathrm{d}}$ \\
\hline & Treated & $0.020 \pm 0.0010^{\mathrm{c}}$ & $0.016 \pm 0.0008^{\mathrm{ab}}$ & $0.020 \pm 0.0010^{\mathrm{c}}$ & $0.015 \pm 0.0008^{\mathrm{a}}$ \\
\hline \multirow{2}{*}{$\mathrm{Cu}\left(\mathrm{mg} \mathrm{L}^{-1}\right)$} & Raw & $0.021 \pm 0.0011^{\mathrm{d}}$ & $0.025 \pm 0.0025^{\mathrm{ef}}$ & $0.024 \pm 0.0012^{\mathrm{e}}$ & $0.027 \pm 0.0014^{\mathrm{g}}$ \\
\hline & Treated & $0.004 \pm 0.0002^{\mathrm{a}}$ & $0.004 \pm 0.0002^{\mathrm{a}}$ & $0.010 \pm 0.0005^{\mathrm{c}}$ & $0.005 \pm 0.0003^{\mathrm{ab}}$ \\
\hline \multirow{2}{*}{$\mathrm{Cr}\left(\mathrm{mg} \mathrm{L}^{-1}\right)$} & Raw & $0.005 \pm 0.0003^{c}$ & $0.005 \pm 0.0005^{\mathrm{c}}$ & $0.005 \pm 0.0003^{\mathrm{c}}$ & $0.006 \pm 0.0003^{\mathrm{d}}$ \\
\hline & Treated & $0.002 \pm 0.0001^{\mathrm{a}}$ & $0.002 \pm 0.0001^{\mathrm{a}}$ & $0.002 \pm 0.0001^{\mathrm{a}}$ & $0.003 \pm 0.0002^{\mathrm{b}}$ \\
\hline \multirow{2}{*}{$\mathrm{Co}\left(\mathrm{mg} \mathrm{L}^{-1}\right)$} & Raw & $0.020 \pm 0.0010^{\mathrm{d}}$ & $0.020 \pm 0.0020^{\mathrm{d}}$ & $0.024 \pm 0.0012^{\mathrm{e}}$ & $0.020 \pm 0.0010^{\mathrm{d}}$ \\
\hline & Treated & $0.009 \pm 0.0005^{\mathrm{ab}}$ & $0.010 \pm 0.0005^{\mathrm{c}}$ & $0.010 \pm 0.0005^{\mathrm{c}}$ & $0.008 \pm 0.0004^{\mathrm{a}}$ \\
\hline \multirow{2}{*}{$\mathrm{Cd}\left(\mathrm{mg} \mathrm{L}^{-1}\right)$} & Raw & $0.040 \pm 0.0020^{\mathrm{f}}$ & $0.030 \pm 0.0030^{\mathrm{e}}$ & $0.026 \pm 0.0013^{\mathrm{d}}$ & $0.022 \pm 0.0011^{\mathrm{c}}$ \\
\hline & Treated & $0.003 \pm 0.0002^{\mathrm{a}}$ & $0.004 \pm 0.0002^{\mathrm{ab}}$ & $0.004 \pm 0.0002^{\mathrm{ab}}$ & $0.003 \pm 0.0002^{\mathrm{a}}$ \\
\hline \multirow{2}{*}{$\mathrm{Ni}\left(\mathrm{mg} \mathrm{L}^{-1}\right)$} & Raw & $0.018 \pm 0.0009^{\mathrm{e}}$ & $0.021 \pm 0.0011^{\mathrm{fg}}$ & $0.022 \pm 0.0022^{\mathrm{h}}$ & $0.020 \pm 0.0020^{\mathrm{f}}$ \\
\hline & Treated & $0.008 \pm 0.0004^{\mathrm{c}}$ & $0.007 \pm 0.0004^{\mathrm{ab}}$ & $0.006 \pm 0.0003^{\mathrm{a}}$ & $0.013 \pm 0.0007^{\mathrm{d}}$ \\
\hline \multirow{3}{*}{$\mathrm{Pb}\left(\mathrm{mg} \mathrm{L}^{-1}\right)$} & Raw & $0.021 \pm 0.0011^{\mathrm{d}}$ & $0.022 \pm 0.0011^{\mathrm{de}}$ & $0.022 \pm 0.0022^{\mathrm{de}}$ & $0.019 \pm 0.0019^{c}$ \\
\hline & Treated & $0.005 \pm 0.0003^{\mathrm{ab}}$ & $0.004 \pm 0.0002^{\mathrm{a}}$ & $0.004 \pm 0.0002^{\mathrm{a}}$ & $0.004 \pm 0.0002^{\mathrm{a}}$ \\
\hline & & Phytoplankton & & & \\
\hline \multirow{2}{*}{$\mathrm{Fe}\left(\mathrm{mg} \mathrm{L}^{-1}\right)$} & Raw & $0.910 \pm 0.0455^{\mathrm{d}}$ & $1.020 \pm 0.0510^{\mathrm{g}}$ & $0.980 \pm 0.0490^{\mathrm{f}}$ & $0.950 \pm 0.0475^{\mathrm{e}}$ \\
\hline & Treated & $0.320 \pm 0.0160^{\mathrm{c}}$ & $0.320 \pm 0.0160^{\mathrm{c}}$ & $0.250 \pm 0.0125^{\mathrm{a}}$ & $0.270 \pm 0.0135^{\mathrm{ab}}$ \\
\hline \multirow{2}{*}{$\operatorname{Mn}\left(\mathrm{mg} \mathrm{L}^{-1}\right)$} & Raw & $0.550 \pm 0.0275^{\mathrm{e}}$ & $0.450 \pm 0.0225^{\mathrm{d}}$ & $0.600 \pm 0.0180^{g}$ & $0.560 \pm 0.0280^{\mathrm{ef}}$ \\
\hline & Treated & $0.090 \pm 0.0500^{\mathrm{b}}$ & $0.090 \pm 0.0045^{\mathrm{b}}$ & $0.160 \pm 0.0080^{c}$ & $0.076 \pm 0.0038^{a}$ \\
\hline \multirow{2}{*}{$\mathrm{Zn}\left(\mathrm{mg} \mathrm{L}^{-1}\right)$} & Raw & $0.350 \pm 0.0175^{\mathrm{g}}$ & $0.300 \pm 0.0150^{\mathrm{f}}$ & $0.280 \pm 0.0140^{\mathrm{e}}$ & $0.280 \pm 0.0140^{\mathrm{e}}$ \\
\hline & Treated & $0.050 \pm 0.0045^{\mathrm{ab}}$ & $0.045 \pm 0.0023^{\mathrm{a}}$ & $0.080 \pm 0.0040^{\mathrm{d}}$ & $0.070 \pm 0.0035^{\mathrm{c}}$ \\
\hline \multirow{2}{*}{$\mathrm{Cu}\left(\mathrm{mg} \mathrm{L}^{-1}\right)$} & Raw & $0.110 \pm 0.0055^{\mathrm{e}}$ & $0.160 \pm 0.0160^{f}$ & $0.200 \pm 0.0100^{\mathrm{g}}$ & $0.230 \pm 0.0115^{\mathrm{h}}$ \\
\hline & Treated & $0.020 \pm 0.0025^{\mathrm{b}}$ & $0.016 \pm 0.0008^{\mathrm{a}}$ & $0.050 \pm 0.0025^{\mathrm{d}}$ & $0.028 \pm 0.0014^{\mathrm{c}}$ \\
\hline \multirow{2}{*}{$\mathrm{Cr}\left(\mathrm{mg} \mathrm{L}^{-1}\right)$} & Raw & $0.025 \pm 0.0013^{\mathrm{d}}$ & $0.030 \pm 0.0030^{\mathrm{e}}$ & $0.033 \pm 0.0017^{\mathrm{f}}$ & $0.036 \pm 0.0018^{\mathrm{g}}$ \\
\hline & Treated & $0.012 \pm 0.0010^{\mathrm{ab}}$ & $0.010 \pm 0.0005^{\mathrm{a}}$ & $0.010 \pm 0.0005^{\mathrm{a}}$ & $0.018 \pm 0.0009^{c}$ \\
\hline \multirow{2}{*}{$\mathrm{Co}\left(\mathrm{mg} \mathrm{L}^{-1}\right)$} & Raw & $0.120 \pm 0.0060^{\mathrm{c}}$ & $0.120 \pm 0.0120^{\mathrm{c}}$ & $0.210 \pm 0.0105^{\mathrm{c}}$ & $0.180 \pm 0.0090^{\mathrm{d}}$ \\
\hline & Treated & $0.050 \pm 0.0006^{\mathrm{a}}$ & $0.070 \pm 0.0035^{\mathrm{b}}$ & $0.070 \pm 0.0035^{\mathrm{b}}$ & $0.050 \pm 0.0025^{\mathrm{a}}$ \\
\hline \multirow{2}{*}{$\mathrm{Cd}\left(\mathrm{mg} \mathrm{L}^{-1}\right)$} & Raw & $0.250 \pm 0.0125^{\mathrm{f}}$ & $0.200 \pm 0.0200^{\mathrm{d}}$ & $0.220 \pm 0.0110^{\mathrm{e}}$ & $0.190 \pm 0.0095^{\mathrm{c}}$ \\
\hline & Treated & $0.020 \pm 0.0025^{\mathrm{a}}$ & $0.020 \pm 0.0010^{\mathrm{a}}$ & $0.021 \pm 0.0011^{\mathrm{ab}}$ & $0.020 \pm 0.0010^{\mathrm{a}}$ \\
\hline \multirow{2}{*}{$\mathrm{Ni}\left(\mathrm{mg} \mathrm{L}^{-1}\right)$} & Raw & $0.090 \pm 0.0045^{\mathrm{e}}$ & $0.120 \pm 0.0060^{\mathrm{f}}$ & $0.150 \pm 0.0150^{\mathrm{h}}$ & $0.140 \pm 0.0140^{\mathrm{g}}$ \\
\hline & Treated & $0.040 \pm 0.0010^{\mathrm{bc}}$ & $0.022 \pm 0.0011^{\mathrm{a}}$ & $0.039 \pm 0.0020^{\mathrm{b}}$ & $0.050 \pm 0.0025^{\mathrm{d}}$ \\
\hline \multirow{2}{*}{$\mathrm{Pb}\left(\mathrm{mg} \mathrm{L}^{-1}\right)$} & Raw & $0.180 \pm 0.0090^{\mathrm{g}}$ & $0.170 \pm 0.0085^{\mathrm{f}}$ & $0.160 \pm 0.0320^{\mathrm{e}}$ & $0.150 \pm 0.0300^{\mathrm{d}}$ \\
\hline & Treated & $0.024 \pm 0.0020^{\mathrm{bc}}$ & $0.020 \pm 0.0010^{\mathrm{a}}$ & $0.023 \pm 0.0012^{\mathrm{b}}$ & $0.024 \pm 0.0012^{\mathrm{bc}}$ \\
\hline
\end{tabular}

The effect of the main factors (water teatment and season) and their interaction on THMs in raw and treated water was significant $(\mathrm{P}<0.05)$ as shown in Table 6 . The effect of water treatment was stronger (with higher $\mathrm{F}$ ratio) than that of a season for all tested THMs. The present results showed that high values of THMs in water were during summer, whereas low concentrations were during winter, with an increase in treated water. The water treatment exhibited its maximum efficiency in winter. THMs specification shows that their presence in both raw and treated water was in the order: chloroform > dichlorobromomethane > dibromochloromethane.

As shown in Fig. 1, chloroform concentrations in raw and treated water was in the range of $2.79-$ $17.43 \mathrm{mg} \mathrm{L}^{-1}$ and 18.42 - $69.75 \mathrm{mg} \mathrm{L}^{-1}$, respectively. The results showed significant variations in dichlorobromomethane in raw and treated water $(\mathrm{P}<0.05)$ ranging from $1.52 \mathrm{mg}$ $\mathrm{L}^{-1}$ (in raw water during winter) to $48.36 \mathrm{mg} \mathrm{L}^{-1}$ (in treated water during summer). The maximum concentration of dibromochloromethane was $25.98 \mathrm{mg} \mathrm{L}^{-1}$ in treated water during summer. Moreover, THMs correlated negatively with nutrients in both the native and treated water. 
Table 5. Pearson's Correlation between physicochemical parameters and season at intake and output of Kafr ElShinawy drinking-water treatment plant - Damietta.

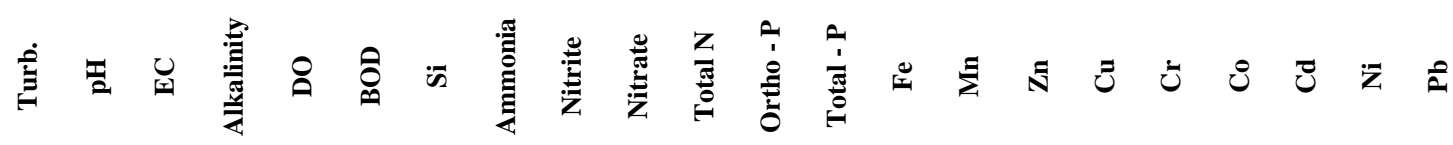

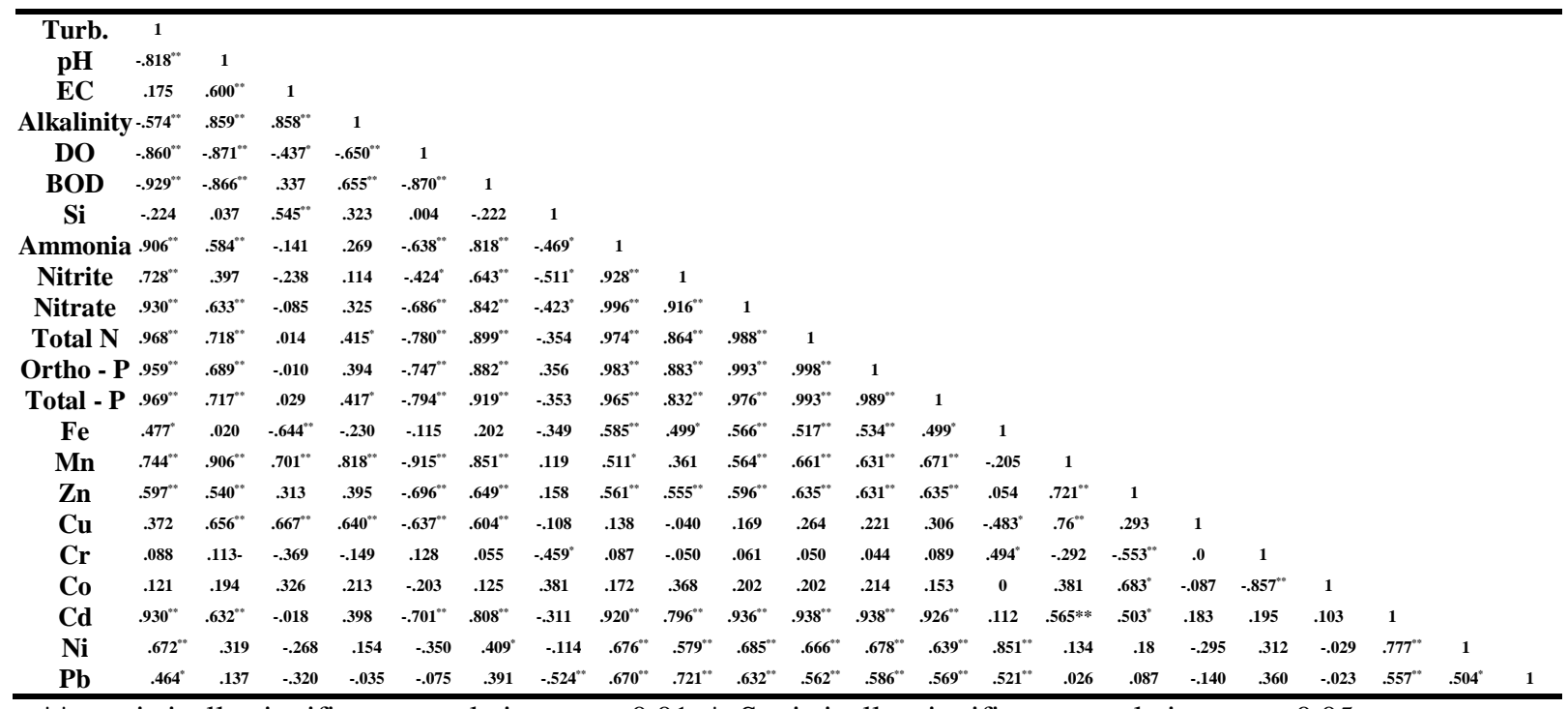

**. statistically significant correlation at $\mathrm{p}<0.01$, *. Statistically, significant correlation at $\mathrm{p}<0.05$.

Table 6. Two- way ANOVA showing the effect of the main factors (water treatment and seasons) and their interaction on Chlorophyll-a of phytoplankton, THMs levels, phytoplankton diversity, and microcystin concentrations in water at Kafr El-Shinawy drinking-water treatment plant - Damietta.

\begin{tabular}{|c|c|c|c|c|c|c|c|}
\hline $\begin{array}{c}\text { Variable and treatment } \\
\text { of variation }\end{array}$ & df & $\mathrm{F}$ & $\mathrm{P}$ & $\begin{array}{l}\text { Variable and treatment of } \\
\text { variation }\end{array}$ & df & $\mathrm{F}$ & $\mathrm{P}$ \\
\hline \multicolumn{4}{|l|}{ Chloroform } & \multicolumn{4}{|c|}{ Bacillariophyta (cell number) } \\
\hline Water treatment & 1 & 17443729 & 0.000 & Water treatment & 1 & 116079.7 & 0.0000 \\
\hline Season & 3 & 4172580 & 0.000 & Season & 3 & 881887.5 & 0.000 \\
\hline Water treatment $\times$ season & 3 & 1427454 & 0.000 & Water treatment $\times$ season & 3 & 280940.1 & 0.000 \\
\hline \multicolumn{4}{|l|}{ Dichlorobromomethane } & \multicolumn{4}{|c|}{ Intramicrocystin concentrations } \\
\hline Water treatment & 1 & 719773777 & 0.000 & Water treatment & 1 & 2910.82 & 0.0000 \\
\hline Season & 3 & 190202800 & 0.000 & Season & 3 & 137.260 & 0.000 \\
\hline Water treatment $\times$ season & 3 & 63348845 & 0.000 & Water treatment $\times$ season & 3 & 134.908 & 0.000 \\
\hline \multicolumn{4}{|l|}{ Dibromochloromethane } & \multicolumn{4}{|c|}{ Extramicrocystin concentrations } \\
\hline Water treatment & 1 & 195832036 & 0.000 & Water treatment & 1 & 11184.8 & 0.0000 \\
\hline Season & 3 & 50480612 & 0.000 & Season & 3 & 2138.21 & 0.000 \\
\hline Water treatment $\times$ season & 3 & 16022276 & 0.000 & Water treatment $\times$ season & 3 & 194.464 & 0.000 \\
\hline \multicolumn{4}{|l|}{ Cyanophyta (cell number) } & \multicolumn{4}{|c|}{ Phytoplankton chlorophyll-a } \\
\hline Water treatment & 1 & 10630415 & 0.000 & Water treatment & 1 & 22814.1 & 0.000 \\
\hline Season & 3 & 1693782 & 0.000 & Season & 3 & 5920.19 & 0.000 \\
\hline Water treatment $\times$ season & 3 & 1461754 & 0.000 & Water treatment $\times$ season & 3 & 4592.06 & 0.000 \\
\hline \multicolumn{8}{|l|}{ Chlorophyta (cell number) } \\
\hline Water treatment & 1 & 2898449 & 0.000 & & & & \\
\hline Season & 3 & 4172580 & 0.000 & & & & \\
\hline Water treatment $\times$ season & 3 & 6563210 & 0.000 & & & & \\
\hline
\end{tabular}



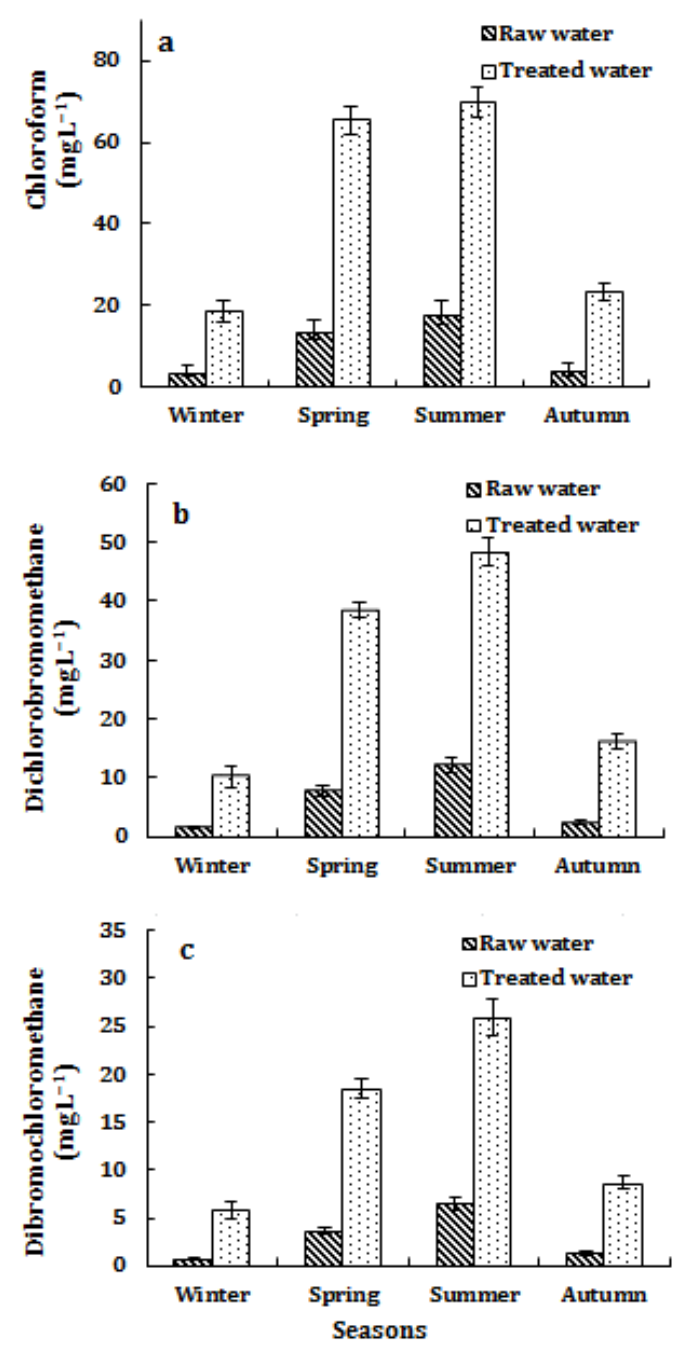

Fig. 1 Concentrations of chloroform (a), dichlorobromomethane (b), and dibromochloromethane (c) in raw and treated water of Kafr El-Shinawy drinking-water treatment plant Damietta. Values are means of three replicates \pm SE.

\subsection{Phytoplankton composition}

Three phytoplankton groups were found in raw and treated waters, viz. Cyanophyta, Chlorophyta and Bacillariophyta. The phytoplankton density in treated water was much less than those in raw water. The effect of the main factors (water treatment and season) and their interaction on the phytoplankton community at the study area was significant $(\mathrm{P}$ $<0.05)$ as shown in Table 6 . The effect of water treatment was stronger (with higher $\mathrm{F}$ ratio) than that of a season for only cell number of Chlorophyta, meanwhile, the effect of season was stronger on Cyanophyta and Bacillariophyta.

The phytoplankton community of raw water was composed mainly from Cyanophyta which contributed up to $67.8 \%$ of the total cell number during spring, summer (91.74\%), autumn $(69.75 \%)$, winter $(14.04 \%)$; and followed by Bacillariophyta, which represents $16.48 \%$ during spring, summer (4.96\%), autumn $(25.70 \%)$, and winter $(77.93 \%)$. Meanwhile, Chlorophyta in raw water represents $15.75 \%$ of the total cell number during spring, summer (3.31\%), autumn $(4.55 \%)$, winter $(8.03 \%)$. In treated water, Chlorophyta was the predominant phytoplankton group which contributed $61.95 \%$ during winter, spring $(82.35 \%)$ and autumn $(22.58 \%)$ of the total cell number, with no detection during summer (Fig. 2). Cyanophyta ranked the second position of dominance with cell number of $13.53 \%$ during spring, (77.78\%) during summer, and (48.39\%) during autumn of the total cell number with no detection during winter. While Bacillariophyta in treated water represents $4.12 \%$ during spring, summer $(22.22 \%)$, autumn (29.03\%), and winter $(38.05 \%)$.

The maximum cell numbers of phytoplankton was found in raw water during summer $(55.5 \times$ $10^{7}$ cell $\left.\mathrm{L}^{-1}\right)$. The species composition of raw water (47 taxa) was richer than that of treated water (only 15 taxa). During winter, Oscillatoria limnetica was predominated in raw water (98.5\% total phytoplankton). Meanwhile, Microcystis aeruginosa predominated during summer $(57.5 \%)$. Other Cyanophyta species also coexisted but in low numbers (Table 7). Pediastrum simplex was the predominant Chlorophyta in raw water throughout the year. In raw water, Melosira granulate predominated Bacillariophyta during winter and autumn while, Cyclotella meneghiniana and Diatoma elongatum were the predominant Bacillariophyta during spring and summer, respectively. In treated water, some Chlorophyta and Bacillariophyta coexisted in low numbers.

Pearson's correlation coefficient revealed that the composition of phytoplankton community depends on the physicochemical parameters of water, which in turn depends on water treatment and seasons. As shown in Table 8, a significant positive correlation was reported between Cyanophyta cell numbers and concentration of total nitrogen $(\mathrm{r}=0.419, \mathrm{P}<0.05)$, and total phosphorus $(\mathrm{r}=0.456, \mathrm{P}<0.05)$, and between Bacillariophyta cell numbers and both silica $(\mathrm{r}=$ $0.356, \mathrm{P}<0.01)$ and nitrite $(\mathrm{r}=.580, \mathrm{P}<0.01)$. 
Table 7. Seasonally variation in the cell number (cell $\left.\times 10^{5} \mathrm{~L}^{-1}\right)$ of the different phytoplankton groups at the intake and output of Kafr El-Shinawy drinking-water treatment plant - Damietta.

\begin{tabular}{|c|c|c|c|c|c|c|c|c|}
\hline \multirow{2}{*}{ Phytoplankton group } & \multicolumn{2}{|c|}{ Winter } & \multicolumn{2}{|c|}{ Spring } & \multicolumn{2}{|c|}{ Summer } & \multicolumn{2}{|c|}{ Autumn } \\
\hline & Intake & Output & Intake & Output & Intake & Output & Intake & Output \\
\hline \multicolumn{9}{|l|}{ Cyanophyta } \\
\hline Anabaena circinalis & - & - & 116 & - & 188 & - & 32 & - \\
\hline A. variabilis & - & - & 112 & - & 178 & - & 28 & - \\
\hline A. constricta & - & - & 97 & - & 168 & - & 23 & - \\
\hline Aphanizomenon flos aquae & 5 & - & 1485 & - & 3919 & - & 860 & - \\
\hline Chroococcus limneticus & - & - & 1182 & - & 1867 & - & 885 & - \\
\hline Coelosphaerium kuetzinglanum & - & - & 30 & - & 80 & - & 10 & - \\
\hline Gloeocapsa aeruginosa & - & - & 1185 & 0.06 & 2441 & 0.2 & 466 & 0.05 \\
\hline Gomphosphaeria lacustris & 6 & - & 1566 & - & 3958 & - & 809 & - \\
\hline Merismopedia glauca & 4 & - & 606 & - & 971 & - & 30 & - \\
\hline M. elegans & - & - & 499 & - & 602 & - & 25 & - \\
\hline M. incerta & - & - & 456 & - & 872 & - & 28 & - \\
\hline Microcystis aeruginosa & 10 & - & 5828 & 0.17 & 29314 & 0.5 & 10416 & 0.1 \\
\hline Nostoc linckia & - & - & 222 & - & 973 & - & 63 & - \\
\hline N. spongiaeforme & - & - & 205 & - & 932 & - & 65 & - \\
\hline N. punctiforme & 3 & - & 1800 & - & 1600 & - & 660 & - \\
\hline Oscillatoria agardhii & - & - & 800 & - & 2000 & - & 1200 & - \\
\hline O. limnetica & 1900 & - & 700 & - & 200 & - & 300 & - \\
\hline Phormidium corium & - & - & 419 & - & 693 & - & 250 & - \\
\hline \multicolumn{9}{|l|}{ Chlorophyta } \\
\hline Actinastrum hantzschii & 44 & - & 200 & - & 133 & - & 88 & - \\
\hline Ankistrodesmus angustus & 50 & - & 140 & - & 40 & - & 31 & - \\
\hline Botryococcus braunii & 31 & - & 180 & - & 77 & - & 37 & - \\
\hline Chlamydomonas spp. & 52 & - & 171 & - & 93 & - & 83 & - \\
\hline Chlorella vulgaris & 31 & - & 154 & 0.1 & 96 & - & 53 & 0.07 \\
\hline Coelastrum microporum & 36 & - & 161 & - & 81 & - & 45 & - \\
\hline Dictyosphaerium pulchellum H. C. Wood & 109 & - & 592 & - & 157 & - & 33 & - \\
\hline Oocystis marssonii & 45 & - & 393 & - & 236 & - & 67 & - \\
\hline Pandorina morum & 17 & - & 180 & - & 100 & - & 80 & - \\
\hline Pediastrum clathratum & 10 & - & 164 & - & 90 & - & 56 & - \\
\hline P. duplex & 65 & - & 186 & - & 133 & - & 129 & - \\
\hline P. simplex & 510 & 0.3 & 1078 & 0.6 & 393 & - & 273 & - \\
\hline Scenedesmus dimorphus & 37 & 0.4 & 187 & 0.7 & 80 & - & 10 & - \\
\hline Staurastrum rotula Nordstedt & 45 & - & 106 & - & 83 & - & 59 & - \\
\hline Ulothrix subitllssima & 20 & - & 130 & - & 44 & - & 10 & - \\
\hline \multicolumn{9}{|l|}{ Bacillariophyta } \\
\hline Amphora coffeaeformis & 157 & - & 69 & - & 72 & - & 115 & $\overline{-}$ \\
\hline Cyclotella meneghiniana & 1827 & - & 783 & - & 347 & - & 907 & - \\
\hline Cyclotella spp. & 600 & 0.2 & 504 & - & 302 & - & 405 & - \\
\hline Diatoma elongatum & 830 & - & 207 & - & 377 & - & 420 & - \\
\hline Fragilaria capucina & 183 & - & 37 & - & 38 & - & 127 & - \\
\hline F. cortonensis & 242 & - & 72 & - & 10 & - & 128 & - \\
\hline Melosira granulata & 1931 & - & 688 & - & 370 & - & 923 & - \\
\hline Navicula radiosa & 923 & - & 243 & - & 253 & 0.2 & 300 & - \\
\hline Nitzschia palea & 200 & - & 104 & 0.07 & 0 & - & 191 & 0.09 \\
\hline N. vermicularis & 631 & 0.03 & 301 & - & 246 & - & 522 & - \\
\hline Stephanodiscus dubius & 1502 & - & 404 & - & 250 & - & 805 & - \\
\hline Synedra acus & 706 & 0.2 & 480 & - & 340 & - & 544 & - \\
\hline S. ulna & 800 & - & 250 & - & 120 & - & 480 & - \\
\hline S. gracillies & 166 & - & 67 & - & 29 & - & 83 & - \\
\hline
\end{tabular}



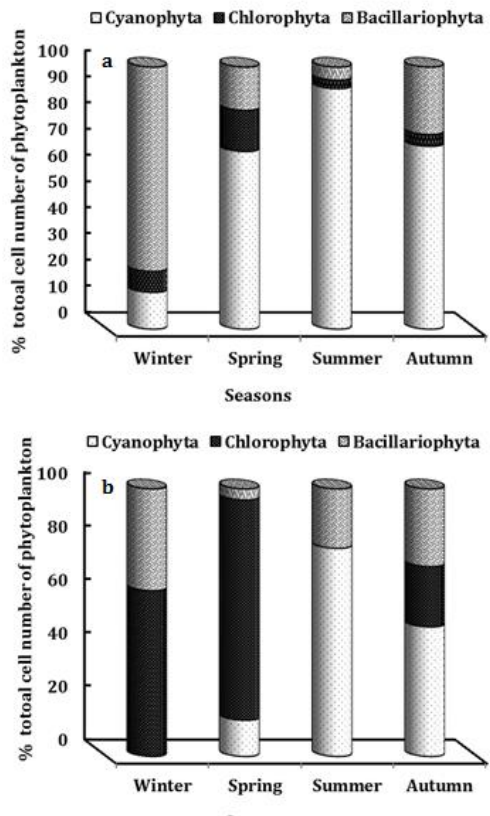

Fig. 2 Seasonal variations in percentage of cell numbers of different phytoplankton groups in raw (a) and treated (b) water of Kafr El-Shinawy treatment plant - Damietta.

\section{Intracellular and extracellular microcystins}

The effect of the main factors (water treatment and season) and their interaction on the levels of intracellular and extracellular microcystin was significant $(\mathrm{P}<0.05)$ with a higher effect of water treatment (higher $\mathrm{F}$ ratio) than that of season (Table 6). Both intracellular and extracellular (dissolved) microcystins recorded their higher concentrations during summer. Throughout the study period, the intracellular microcystin levels were lower in treated water than in raw water. In raw water, the lowest intracellular microcystin was obtained during winter $\left(0.71 \mu \mathrm{g} \mathrm{L}^{-1}\right)$ while the highest concentration was $1.7 \mu \mathrm{g} \mathrm{L}^{-1}$ during summer (Table 9). The maximum concentrations of dissolved microcystins in raw water $(1.30 \mu \mathrm{g}$ $\left.\mathrm{L}^{-1}\right)$ was lower than that in treated water $(2.01$ $\mu \mathrm{g} \mathrm{L}^{-1}$ ) during summer. Also, the minimum concentrations of dissolved microcystins in raw water $\left(0.56 \mu \mathrm{g} \mathrm{L}^{-1}\right)$ during winter was lower than that in treated water $\left(1.00 \mu \mathrm{g} \mathrm{L}^{-1}\right)$ during autumn.

\section{Biochemical composition of the predominant phytoplankton species in raw and treated water}

Biochemical constituents of the predominant species in raw water; Oscillatoria limnetica and
Microcystis aeruginosa were estimated during winter and summer, respectively (Fig. 3). Protein, lipid and carbohydrates were significantly different between the two species. M. aeruginosa was richer in protein $(47 \% \mathrm{DW})$ and lipid (4.28\% DW) than O. limnetica (40.4 and $3.2 \% \mathrm{DW}$, respectively). By contrast, total carbohydrate were higher in O. limnetica (29.6 $\% \mathrm{DW})$ than M. aeruginosa (21.6\% DW).

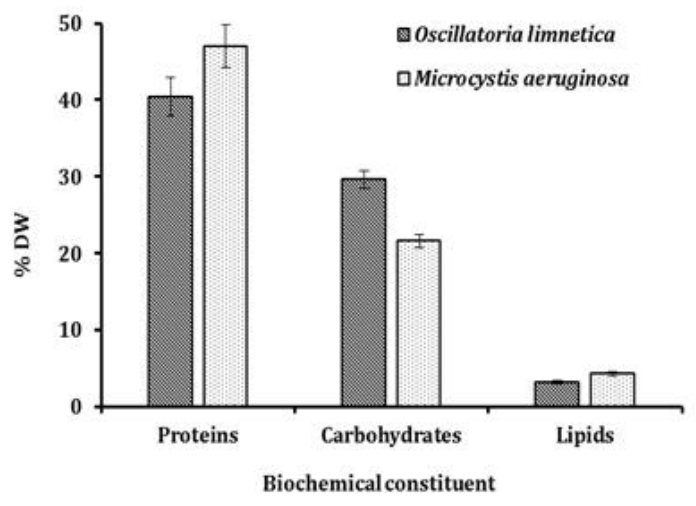

Fig. 3 Variations in concentrations of some biochemical constituents (\% DW) of Oscillatoria limnetica in winter and Microcystis aeruginosa in summer, respectively in raw water at Kafr ElShinawy drinking-water treatment plant - Damietta. Values are means of three replicates \pm SE.

The effect of the main factors (water treatment and season) and their interaction on chlorophyll-a content of phytoplankton of raw and treated water was very highly significant $(\mathrm{P}$ $<0.05$ ) with a higher effect of water treatment than that of a season (Table 6). The chlorophylla content in phytoplankton was significantly higher in raw water than in treated water during the study period $(\mathrm{P}<0.01)$, particularly during spring (Fig. 4). Chlorophyll-a content was generally highest during summer $\left(1.42 \mu \mathrm{g} \mathrm{L}^{-1}\right)$, followed by spring $\left(1.21 \mu \mathrm{g} \mathrm{L}^{-1}\right)$, while the lowest values were during winter $\left(0.04 \mu \mathrm{g} \mathrm{L}^{-1}\right)$.

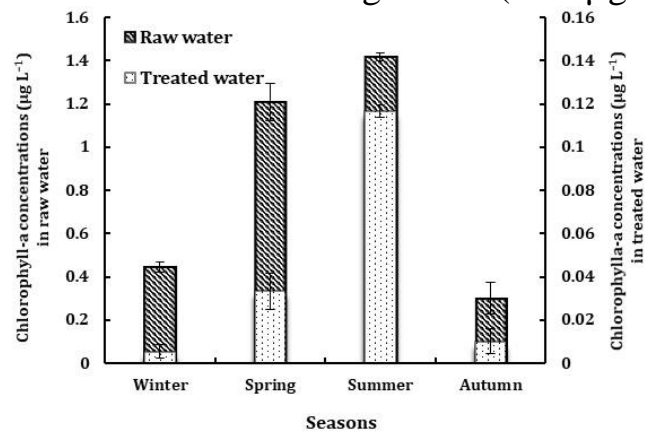

Fig. 4 Phytoplankton chlorophyll-a contents $\left(\mu \mathrm{g} \mathrm{L}^{-1}\right)$ in raw and treated water of Kafr El-Shinawy drinking-water treatment plant - Damietta. Values are means of three replicates \pm SE. 
Table 8. Pearson's correlation coefficients between trophic status of water, THMs levels, phytoplankton diversity, and microcystin concentration at the intake and output of Kafr El-Shinawy drinking-water treatment plant Damietta.

$$
\text { 苛 }
$$

\begin{tabular}{|c|c|c|c|c|c|c|c|c|c|c|c|c|c|c|c|}
\hline Silica & 1 & & & & & & & & & & & & & & \\
\hline Ammonia & $-.469^{*}$ & 1 & & & & & & & & & & & & & \\
\hline Nitrite & $-.511^{*}$ & $.928^{* *}$ & 1 & & & & & & & & & & & & \\
\hline Nitrate & $-.423^{*}$ & $.996^{* *}$ & $.916^{* *}$ & 1 & & & & & & & & & & & \\
\hline Total N & -.354 & $.974^{* *}$ & $.864^{* *}$ & $.988^{* *}$ & 1 & & & & & & & & & & \\
\hline Ortho P & -.356 & $.983^{* *}$ & $.883^{* *}$ & $.993^{* *}$ & $.998^{* *}$ & 1 & & & & & & & & & \\
\hline Total P & -.353 & $.965^{* *}$ & $.832^{* *}$ & $.976^{* *}$ & $.993^{* *}$ & $.989^{* *}$ & 1 & & & & & & & & \\
\hline Chloroform & $.466^{*}$ & $-.733^{* *}$ & $-.694^{* *}$ & $-.727^{* *}$ & $-.708^{* *}$ & $-.704^{* *}$ & $-.692^{* *}$ & 1 & & & & & & & \\
\hline $\begin{array}{c}\text { Dichlorobromometh } \\
\text { ane }\end{array}$ & $.450^{*}$ & $-.719^{* *}$ & $-.682^{* *}$ & $-.712^{* *}$ & $-.691^{* *}$ & $-.689^{* *}$ & $-.675^{* *}$ & $.993^{* *}$ & 1 & & & & & & \\
\hline $\begin{array}{c}\text { Dibromochlorometh } \\
\text { ane }\end{array}$ & $.465^{*}$ & $-.727^{* *}$ & $-.688^{* *}$ & $-.719^{* *}$ & $-.697^{* *}$ & $-.696^{* *}$ & $-.680^{* *}$ & $.981^{* *}$ & $.997^{* *}$ & 1 & & & & & \\
\hline Cyanophyta number & -.062 & .299 & .065 & .331 & $.419^{*}$ & .384 & $.456^{*}$ & -.003 & .080 & .107 & 1 & & & & \\
\hline $\begin{array}{l}\text { Chlorophyta } \\
\text { number }\end{array}$ & .344 & $-.575^{* *}$ & $-.520^{* *}$ & $-.583^{* *}$ & $-.593^{* *}$ & $-.582^{* *}$ & $-.592^{* *}$ & .365 & .260 & .212 & $-.745^{* *}$ & 1 & & & \\
\hline $\begin{array}{c}\text { Bacillariophyta } \\
\text { number }\end{array}$ & $-.356^{* *}$ & .309 & $.580^{* *}$ & .273 & .158 & .194 & .102 & $-.470^{*}$ & $-.454^{*}$ & $-.431^{*}$ & $-.496^{*}$ & -.209 & 1 & & \\
\hline Intramicrocystin & $.838^{* *}$ & .090 & .301 & .209 & .334 & .393 & .376 & .111 & -.097 & -.097 & .382 & -.259 & -.222 & 1 & \\
\hline Extramicrocystin & -.176 & $.535^{* *}$ & $-.841^{* *}$ & $-.816^{* *}$ & $-.821^{* *}$ & $-.768^{* *}$ & $-.781^{* *}$ & $.924^{* *}$ & $.935^{* *}$ & $.942^{* *}$ & .087 & .303 & $.521^{* *}$ & $-.504^{*}$ & 1 \\
\hline
\end{tabular}

**. statistically significant correlation at $\mathrm{p}<0.01, *$. Statistically, significant correlation at $\mathrm{p}<0.05$.

Table 9. Seasonally variations in concentrations $\left(\mu \mathrm{g} \mathrm{L}^{-1}\right)$ of intracellular and extracellular microcystins (Mean \pm $\mathrm{SE}, \mathrm{n}=3$ ) in raw and treated waters at Kafr El-Shinawy drinking-water treatment plant - Damietta.

\begin{tabular}{|c|c|c|c|c|c|}
\hline \multirow{2}{*}{ Microcystin } & \multirow{2}{*}{ Treatment } & \multicolumn{4}{|c|}{ Season } \\
\hline & & Winter & Spring & Summer & Autumn \\
\hline \multirow{2}{*}{ Intracellular microcystin } & Raw & $0.710 \pm 0.0284^{\mathrm{d}}$ & $0.980 \pm 0.0392^{\mathrm{f}}$ & $1.700 \pm 0.0680^{\mathrm{g}}$ & $0.880 \pm 0.0352^{\mathrm{e}}$ \\
\hline & Treated & $0.009 \pm 0.0003^{\mathrm{b}}$ & $0.009 \pm 0.0003^{\mathrm{b}}$ & $0.011 \pm 0.0003^{\mathrm{bc}}$ & $0.003 \pm 0.0001^{\mathrm{a}}$ \\
\hline \multirow{2}{*}{ Extracellular microcystin } & Raw & $0.560 \pm 0.0168^{\mathrm{a}}$ & $0.680 \pm 0.0340^{\mathrm{b}}$ & $1.300 \pm 0.0520^{\mathrm{f}}$ & $0.740 \pm 0.0518^{\mathrm{c}}$ \\
\hline & Treated & $1.210 \pm 0.0242^{\mathrm{e}}$ & $1.780 \pm 0.0890^{\mathrm{g}}$ & $2.010 \pm 0.1005^{\mathrm{h}}$ & $1.000 \pm 0.0400^{\mathrm{d}}$ \\
\hline
\end{tabular}

\section{Discussion}

Evaluation of the efficiency of water treatment regimes, in terms of the alteration in the physicochemical characteristics of water before and after treatment is essential for recommendation of water usage in drinking and othe domestic purposes (Sarkar et al. 2020). The present work revealed that raw water at Kafr El-Shinawy drinking-water treatment plant is highly trophic with high load of nutrients and silica, along with a slightly alkaline $\mathrm{pH}$ and low DO levels. Water temperature was correlated positively with $\mathrm{pH}$ $(\mathrm{r}=0.667, \mathrm{p}<0.01), \mathrm{EC}(\mathrm{r}=0.755, \mathrm{p}<0.01)$, alkalinity $(\mathrm{r}=0.788, \mathrm{p}<0.01)$, and BOD $(\mathrm{r}=$
$0.517, \mathrm{p}<0.01)$. These correlations agreed with that obtained by Sharma et al. (2008) and Shehata and Badr (2010). The intimate correlation between water temperature and $\mathrm{pH}$ is in agreement with Larsen (2013) who reported that temperature changes affect ion concentrations and hence the water $\mathrm{pH}$. Variations in water temperature have been reported to strongly affect the composition, bioactivity, and growth of phytoplankton community (Rasconi et al. 2017).

Water turbidity was significantly correlated with nutrient concentrations (ammonia, nitrite, nitrate, total nitrogen, ortho-P, and total-P) in water. The high turbidity of raw water compared with treated water might be related to high organic pollution of raw water and the efficiency of treatment in water purification. 
Water $\mathrm{pH}$ is an important factor in the aquatic system, that directly affect phytoplankton community. Slight changes in water $\mathrm{pH}$ might be due to biological activity such as photosynthesis and respiration (Nassar et al. 2014). The slight decrease in $\mathrm{pH}$ of treated water below that of raw water was due to the addition of chlorine and alum during treatment processes of raw water (Larsen, 2013).

Dissolved Oxygen (DO) level is an indicator of the water's ability to support a well-balanced aquatic life and acts as an indicator of the trophic status of the water body (George et al. 2012, Salah and El-Moselhy 2015) The increase in DO of treated water above that of raw water might be due to the physicochemical treatment processes of water such as aeration, coagulation, sedimentation, filtration, and addition of oxidative agents. These treatments increased DO and decreased the turbidity of treated water. A significant negative correlation between $\mathrm{DO}$ and water temperatures $(\mathrm{r}=-0.502, \mathrm{p}<0.05)$ was also reported by Shehata and Badr (2010). Low values of DO in raw water during the summer might be attributed to high sewage and agricultural pollution, that enhance microbial growth in raw water.

Low silica concentrations in raw water might be related to the high growth of diatoms, especially during winter. But, the increased silica in treated water can be related to water recycling of reactive silica as a result of disruption and hydrolysis of some diatoms through water treatment in the flocculation basin and during other treatment processes (Shehata and Badr 2010). The extremely low levels of ammonia and nitrite in treated water may be attributed to the oxidation of ammonia and nitrite in the flocculation basin by chlorine. The overall low levels of inorganic nitrogen (ammonia, nitrite, and nitrate) in treated water might be related to their reaction with the chemical reagents during water treatment in the flocculation basin. The pattern of low nutrient level in treated water below raw water, with marked seasonal interaction points to an efficient water treatment regime at the experimental water treatment station.

Some heavy metals are xenobiotics, such as $\mathrm{Pb}$, and $\mathrm{Cd}$; whereas some other heavy metals including $\mathrm{Cu}, \mathrm{Zn}$, and $\mathrm{Cr}$ are essential elements for human body in small quantities, but turn toxic in high doses. In the present study, the low concentrations of heavy metals ( $\mathrm{Fe}, \mathrm{Mn}, \mathrm{Zn}, \mathrm{Cu}$,
$\mathrm{Cr}, \mathrm{Co}, \mathrm{Cd}, \mathrm{Ni}, \mathrm{Pb}$ ) in treated water than in raw water may be related to coagulation and sedimentation processes in treatment basins, in addition to the efficiency of physico-chemical water treatment processes including ion exchange and precipitation. The levels of all the measured heavy metals, especially Mn, Zn, and $\mathrm{Fe}$ were higher in phytoplankton cells than in raw water and treated water. Phytoplankton is widely used as bio-indicator of heavy metal pollution in water bodies. Bioaccumulation capacity of phytoplankton for heavy metals depends on metal type and phytoplankton species. Phytoplankton cells contain different functional groups, including amino, thio, carboxylic, and hydroxo that can interact with heavy metals (Pourkhabbaz et al. 2018). One of the serious problems for human health is high levels of THMs in drinking water that can lead to considerable burden of bladder cancer (Evlampidou et al. 2020). High concentrations of THMs in treated waters were related to the production of THMs as by-products during the chlorination of water (Ivahnenko and Zogorski 2006).

Phytoplankton community can be considered as a bio-indicator for water quality. In the present study, Cyanophyte species predominated phytoplankton in raw water during summer and spring as a result of their optimal growth at high temperatures (Reynolds 1984). The predominance of Bacillariophyta in raw water during winter was in agreement with Ganjian et al. (2010) who concluded that Bacillariophyta growth was favored by the low temperature. High numbers of Chlorophyta in water during the winter might be attributed to dominance of various species of Pediastrum that flourishes in winter months (Cho et al. 2017). In contrast to the present result, Rajagopal et al. (2010) pointed out that the productivity of Chlorophyta increased at high water temperature. High turbidity of water during summer is responsible for the decrease in Chlorophyta growth as it prevents sufficient light required for Chlorophyta growth (Boyd 1990). A negative correlation between Bacillariophyta numbers and silica concentration of water was also reported by Cetin and Sen (1998).

Estimating phytoplankton chlorophyll-a content in water is a direct way of tracking phytoplankton growth and algal blooms (Driver and Justus, 2016). High phytoplankton chlorophyll-a levels indicate the 
high nutrient content of water especially nitrogen and phosphorus. Differences in chlorophyll-a concentration during the study period and according to water treatment reflect changes in phytoplankton numbers in raw and treated water. Similar to cell numbers of Cyanophyta, phytoplankton chlorophyll-a concentrations was not completely depleted after various treatment processes.

The present results are in agreement with Mohamed et al. (2015) that microcystin production increases in accordance with the increase in water temperature and level of nutrients. The existence of higher concentrations of extracellular microcystin in treated water than raw water can be related to the release of the intracellular microcystin in water as a consequence of membrane leakage of cyanobacterial cells through the effect of preoxidant compounds such as chlorine dioxide, ozone, copper sulfate, and chlorine on membrane integrity (Pantelic et al. 2013). Meanwhile, the high levels of extracellular microcystin in treated water occurred at the expense of intracellular microcystin. There were various microcystins variants produced by M. aeruginosa, isolated from the Nile River such as microcystin-LR, microcystin-RR, and microcystin-YR. Moreover, environmental conditions can also indirectly affect microcystin peoduction (Mohamed 2011).

\section{Conclusions}

Phytoplankton composition depends on the changes in physicochemical properties of water as well as the trophic status and pollutant content in water. The optimized physicochemical properties of water and high trophic status increase the phytoplankton growth especially, cyanobacteria to a level of bloom formation. The high growth of cyanobacteria led to production of cyanotoxins with high content of intracellular microcystin and low content of extracellular microcystin in raw water. On the contrary, most of the intracellular microcystins were released in treated water during water treatment processes. Phytoplankton cells control the levels of heavy metals in raw and treated water through their bioaccumulation capacity. Consequently, Heavy metal levels in raw and treated water are less than those in phytoplankton cells. THMs were higher in treated water than in raw water, with marked efficiency of the physicalchemical treatment of water in the flocculation basin. The dissolved microcystin and THMs contents in treated water are higher than the allowable limit.

\section{Acknowledgment}

The authors would like to express their deep thanks and gratitude to all members of the Faculty of Science, Damietta University, Egypt for their valuable advice, guidance, continuous and unlimited support throughout the whole work.

\section{Funding}

This research did not receive any specific grant from funding agencies in the public, commercial, or not-for-profit sectors.

\section{Compliance with ethical standards}

Conflict of interest The authors declare that they have no conflict of interest.

Ethical approval This article does not contain any studies with human participants or animals performed by any of the authors.

\section{References}

AOAC. (2000). Official Method of the Association of Official Analytical Chemists. 17th Edn., AOAC, (pp. 21-447), Washington, DC., USA.

APHA (American Public Health Association). Anonymous. (1996). Standard Methods for the Examination of Water and Wastewater, 17th edn. APHA, Washington, D.C.

Botes, L. (2003). Phytoplankton Identification Catalogue. Saldanha Bay, South Africa, GloBallast monograph No. (7)

Boyd, C. E. (1990). Water Quality in Ponds for Aquaculture. Alabama Agricultural Experiment Site, (pp. 482) Auburn University, Auburn, Alabama.

Cetin, A. K., \& Sen, B. (1998). Diatoms (Bacillariophyta) in the phytoplankton of Keban Reservoir and their seasonal variations. Turkish Journal of Botany, 22(1), 25-33.

Cho, D., Choi, J., Kang, Z. Kim, B., Oh, H., Kim, H., \& Ramanan R. (2017). Microalgal diversity fosters stable biomass productivity in open ponds 
treating wastewater. Scientific Reports, 7, 1979.

Chorus, I., Falconer, I. R., Sala, H. J., \& Bartram, J. (2000). Health risks caused by freshwater cyanobacteria in recreational waters. Journal of Toxicology and Environmental Health, 3, 323347.

Driver, L. J., \& Justus, B. G. (2016). Water-quality effects on phytoplankton species and density and trophic state indices at Big Base and Little Base Lakes, Little Rock Air Force Base, Arkansas, June through august, 2015. Journal of the Arkansas Academy of Science, 70, 16.

Dubois, M., Gilles, K. A., Hamilton, J. K., Rebers, P. A., \& Smith, F. (1956). Colorimetric method for determination of sugars and related substances. Analytical Chemistry, 28, 350-356.

Evlampidou, I., Font-Ribera, L., Rojas-Rueda, D., Gracia-Lavedan, E., Costet, N., Pearce, N., et al. (2020). Trihalomethanes in Drinking Water and Bladder Cancer Burden in the European Union. Environmental Health Perspectives, 128(1), 017001

Ganjian, A., Wan Maznah, W. O., Fazli, H., Vahedi, M., Roohi, A., \& Farabi, S. M. V. (2010). Seasonal and regional distribution of phytoplankton in the southern part of the Caspian Sea. Iranian Journal of Fisheries Sciences, 9(3), 382-401.

George, B. J. I., Kumar, N., \& Kumar, R. N. (2012). Study on the influence of hydro-chemical parameters on phytoplankton distribution along Tapi estuarine area of Gulf of Khambhat, India. Egyptian Journal of Aquatic Research, 38(3), 157-170.

Grasshoff, K. (1975). The Hydrochemistry of Landlocked Basins and Fjords. In: Chemical Oceanography, (pp. 568-574), Academic Press, London.

Harada, K., Ogawa, K., Matsuura, K., Murata, H., Suzuki, M., Watanabe, M. F., Itezono, Y., \& Nakayama, N. (1990). Structural determination of geometrical isomers of microcystins LR and RR from cyanobacteria by two-dimensional NMR spectroscopic techniques. Chemical Research in Toxicology, 3(5), 473-481.

Ivahnenko, T., \& Zogorski, J. S. (2006). Treatments and Occurrence of Chloroform and Other Trihalomethanes in Drinking-Water Supply Wells in the United States, 1986-2001, Scientific Investigations Report No. 2006-5015 (U.S. Department of the Interior and U.S. Geological Survey).

Krammer, K., \& Lange-Bertalot, H. (1986). Bacillariophyceae. Teil: Naviculaceae. Süsswasserflora von Mitteleuropa, (pp. [i]-xvi, [1]-876, 206), Gustav Fischer Verlag, Jena, Germany.

Kumar, A., \& Sahu, R. (2012). Diversity of algae
(Cholorophyceae) in paddy fields of Lalgutwa area, Ranchi, Jharkhand. Journal of Applied Pharmaceutical Science, 2(11), 92-95.

Larsen, D. (2013). Temperature Dependent of the $\mathrm{pH}$ of pure Water. In UC Davis Chem Wiki. Retrieve from

http://chemwiki.ucdavis.edu/Physical_Chemistry/A cids_and_Bases/Aqueous_Solutions/The_pH_S cal e/Temperature _Dependent_of_the_pH_of_pure_Water

Manickam, N., Saravana Bhavan, P., Vijayan, P., \& Sumathi, G. (2012). Phytoplankton species diversity in the Parambikulam-Aliyar irrigational canals (Tamilnadu, India). International Journal of Pharma and Bio Sciences, 3(3), 289 - 300.

Metzener, H., Rau, H., \& Senger, H. (1965). untersuchungen Zur Synchronisierbarkeit einzelener Pigment-Mangel Mutanten Von Chlorella. Planta, 65, 186-194.

Mohamed, Z. A. (2011). Cyanotoxins in Egypt and Saudi Arabia, In: Nriagu JO (Ed) Encyclopedia of environmental health, (pp. 872-880), Burlington, Elsevier.

Mohamed, Z. A., \& Carmichael, W. W. (2000). Seasonal variation in microcystin levels of river Nile water at Sohag City, Egypt. Annales de Limnologie, 36(4), 227-234.

Mohamed, Z. A., Deyab, M. A., Abou-Dobara, M. I., El-Sayed, A. K., \& El-Raghi, W. M. (2015). Occurrence of cyanobacteria and microcystin toxins in raw and treated waters of the Nile River, Egypt: implication for water treatment and human health. Environmental Science and Pollution Research, 22, 11716-11727.

Nassar, M. Z., Mohamed, H. R., Khiray, H. M., \& Rashedy, S. H. (2014). Seasonal fluctuations of phytoplankton community and physico-chemical parameters of the north western part of the Red Sea, Egypt. Egyptian Journal of Aquatic Research, 40(4), 395-403.

Paerl, H. W., \& Otten, T. G. (2013). Harmful cyanobacteria blooms: cause, consequences, and controls. Microbial Ecology, 65, 995-1010.

Pantelic, D., Svircev, Z., Simeunovic, J., Vidovic, M., \& Trajkovic, I. (2013). Cyanotoxins: characteristics, production and degradation routes in drinking water treatment with reference to the situation in Serbia. Chemosphere, 91, 421441.

Pourkhabbaz, H. R., Hedayatzadeh, F., \& Cheraghi, M. (2018). Determination of heavy metals concentration at water treatment sites in Ahwaz and Mollasani using bioindicator. Ecopersia, 6(1), 55-66.

Rajagopal, T., Thangamani, A., \& Archunan, G. (2010). Comparison of physico-chemical parameters and phytoplankton species diversity 
of two perennial ponds in Sattur area, Tamil Nadu. Journal of Environmental Biology, 31(5), 787-794.

Rasconi, S., Winter, K., \& Kainz, M. J. (2017). Temperature increase and fluctuation induce phytoplankton biodiversity loss Evidence from a multi-seasonal mesocosm experiment. Ecology and Evolution, 7(1), 1-11.

Reynolds, C. S. (1984). The ecology of freshwater phytoplankton. Cambridge University Press, Cambridge.

Salah, A. A., \& El-Moselhy, K. M. (2015). Seasonal variations of the physical and chemical properties of seawater at the Northern Red Sea, Egypt. Open journal of ocean and coastal sciences, 2(1), 1-17.

Sarkar, R., Ghosh, A. R., \& Mondal, N. K. (2020). Comparative study on physicochemical status and diversity of macrophytes and zooplanktons of two urban ponds of Chandannagar, WB, India. Applied Water Science, 10, 63.

Sharma, P. D. (2002). Practical Manual: Ecology and Environmental. Rastogi Publications, (pp. 569-572), Meerut, India.

Sharma, S., Savita, D., Jain, P., Shah, K. W., \& Vishwakarma, R. (2008). Statistical evaluation of hydrobiological parameters of Narmada river water at Hoshangabad city, India. Environmental Monitoring and Assessment, 143, 195-202.

Shehata, S. A., \& Badr, S. A. (2010). Water Quality
Changes in River Nile Cairo, Egypt. Journal of Applied Sciences Research, 6(9), 1457-1465.

Sudharsan, S., Seedevi, P., Ramasamy, P., Subhapradha, N., Vairamani, S., \& Shanmugam, A. (2012). Heavy metal accumulation in seaweeds and sea grasses along southeast coast of India. Journal of Chemical and Pharmaceutical Research, 4(9), 4240-4244.

Tikkanen, T. (1986). Kasviplanktonopas. Suomen Luonnonsuojelun Tuki Oy (Italia.), (pp. 278), Helsinki.

U.S. EPA. (1995). Method 551.1: Determination of Chlorination Disinfection Byproducts, Chlorinated Solvents, and Halogenated Pesticides/Herbicides in Drinking Water by Liquid-Liquid Extraction and Gas Chromatography With Electron-Capture Detection. Revision 1.0. Cincinnati, $\mathrm{OH}$.

Vesterkvist, P. S. M., Misiorek, J. O., Spoof, L. E. M., Tiovola, D. M., \& Meriluoto, J. A. O. (2012). Comparative cellular toxicity of hydrophilic and hydrophobic microcystins on caco-2 cells. Toxins, 4, 1008-1023.

Visser, P. M., Verspagen, J. M. H., Sandrini, G., Stal, L. J., Matthijs, H. C. P., Davis, T. W., Paerl, H. W., \& Huisman, J. (2016). How rising CO2 and global warming may stimulate harmful cyanobacterial blooms. Harmful Algae, 54, 145159.
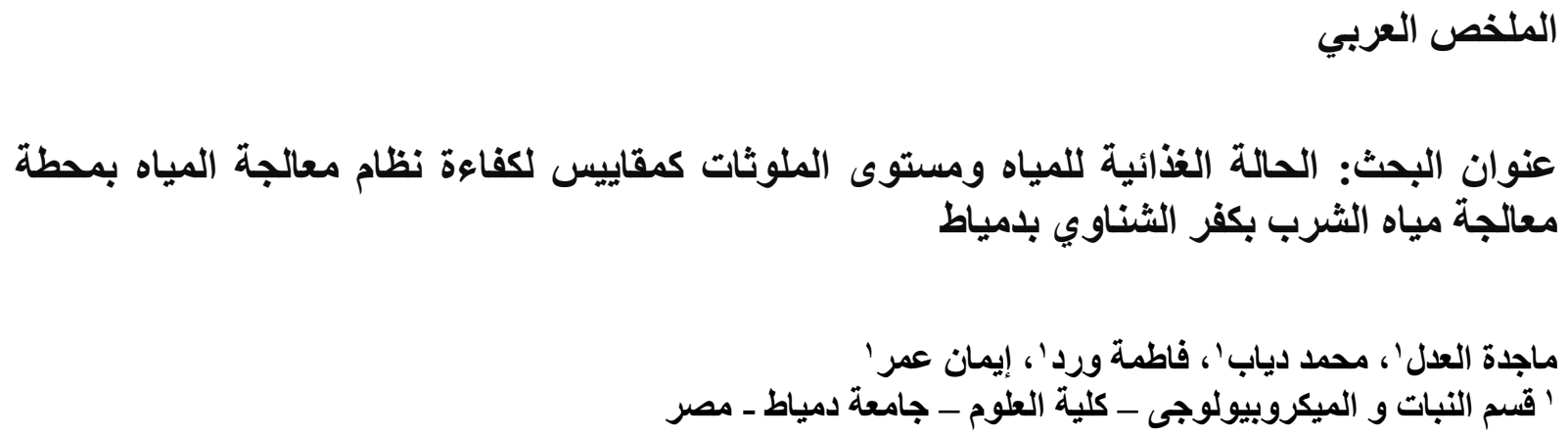

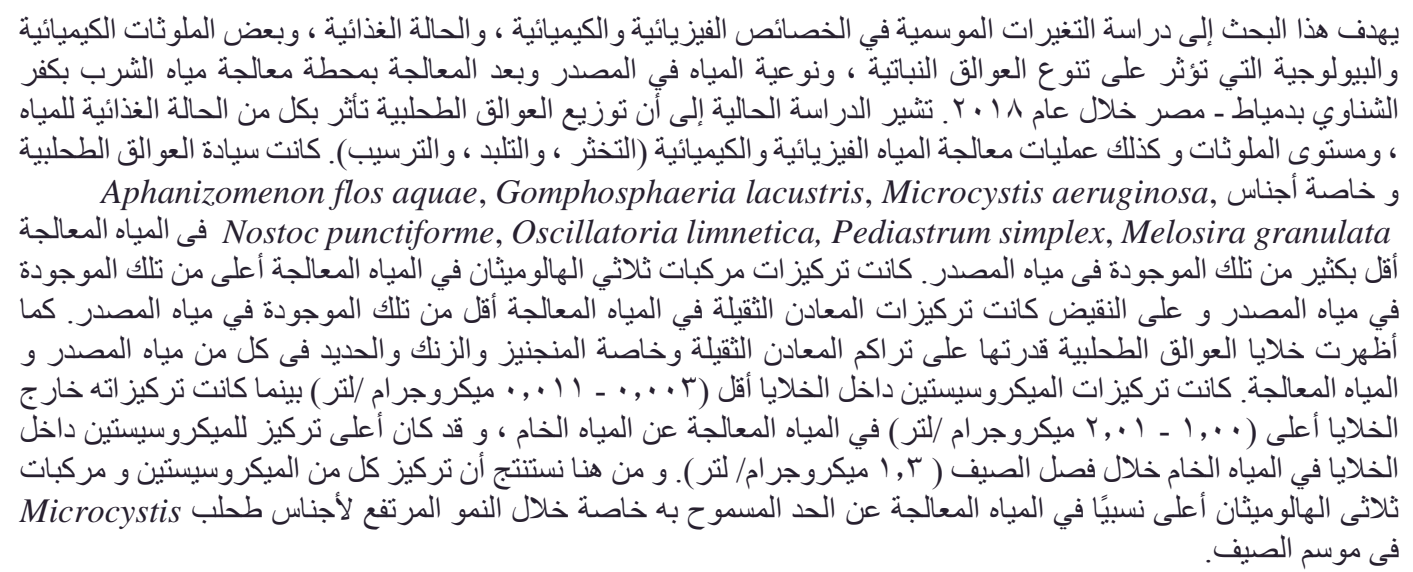

